\title{
A Digital Watermarking Algorithm Based on Chaos in Interpolatory Orthogonal Multiwavelets Domain
}

\author{
Jiang $\mathrm{Li}^{1} \&$ Liu Chen ${ }^{1}$ \\ ${ }^{1}$ School of Science, Hunan University of Technology, Hunan Zhuzhou, China \\ Correspondence: Jiang Li, School of Science, Hunan University of Technology, Hunan Zhuzhou, China. Tel: \\ 86-731-221-833-85. E-mail: jiangli9918@163.com
}

Received: December 12, 2012 Accepted: January 12, 2013 Online Published: March 4, 2013

doi:10.5539/cis.v6n2p31

URL: http://dx.doi.org/10.5539/cis.v6n2p31

This work was supported by the Soft Science Research Project of Hunan Province of China under Grant 2011ZK5008 and by the China National Science Foundation under Grant 10971059

\begin{abstract}
Based on chaotic scrambling, we proposed a digital image watermarking scheme in interpolatory orthogonal multiwavelets transforming domain. At first, the logistic chaotic mapping and arnold transformating are employed to scramble the original watermarking image, then the watermark information is embedded into the middle frequency interpolatory orthogonal multiwavelets transforming coefficients of the image. Experimental results showed that the scheme has high secrecy intensity and ability against the general image processing attack.
\end{abstract}

Keywords: interpolatory orthogonal multiwavelets, arnold transforming, logistic chaotic mapping, digital watermarking

\section{Introduction}

The digital image watermarking technology has become a novel and broad applied prospects' research focus in today's information science field. Usually, the digital image watermark should be required to meet three basic demands (Lu et al., 2006): security, transparency and robustness. It has been proved that the chaotic mapping has the characteristics of higher sensitivity for initial value, stronger security, larger key space and non-periodic. Nowadays, it has many ways to improve the performance of the digital image watermarking algorithm based on chaotic mapping such as taking the chaotic mapping sequence for watermarking signal (Xiang et al., 1999), the watermarking signal being embedded randomly (Gao \& Zhang, 2007), scrambling the watermarking signal and so on (Fan \& Sun, 2006). Commonly, the magic transforming and arnold transforming could be used for scrambling digital image, they also can be used for encrypting digital image, but the shortcomings of the magic transforming and arnold transforming are distinct such as their key space is less, the scrambled matrix is periodic and the scrambled image will be reverted to the original image with many times magic transforming or arnold transforming. The results in the attacker can crack and recovery the watermarking image easily when the watermarking algorithm is public. When employing chaotic mapping for scrambling the digital image only, it will make up a permutation group which results in lower security performance. In order to improve the security performance of the scrambled digital image watermark, one way is to enlarge the key space and overcome the effects of the permutation group, for this purpose the paper combined arnold transforming and logistic chaotic mapping to scramble the meaningful grayscale digital image watermarking. Nowadays, in order to take account of the transparency and robustness of digital image watermarking, the watermarking information are embedded into the image's DCT or DWT transforming coefficients (Jiao \& Ding, 2011).

At present, the digital image watermarking algorithm based on wavelet transforming becoming the focus study of the transforming domain's watermarking algorithm is due to the wavelet transforming is more suitable for human visual system than the DCT transforming in image processing, compatible with most international image processing standards, resisting cutting and lossy compression encoding such as JEPG more effectively (Zhao \& Qi, 2004).

Because of multiwavelets can meet with symmetry, compactly supported, higher order vanishing moments and orthogonality simultaneously (Chui \& Lian, 1996) it has a significant advantage over the wavelet in terms of 
signal processing applications. While the interpolatory orthogonal multiwavelets transforming makes the multiresolution analysis decomposing or coefficients reconstructing to be calculated by their sampled points without of calculating inner product (Yang, 2006), so that it makes the multiwavelets transforming of scalar sequence leaving out prefiltering (Xia et al.,1996), and improving the computational efficiency greatly, which makes the interpolatory orthogonal multiwavelets being popular in some applied fields (Jiang et al., 2009).

The paper proposed a digital image watermarking algorithm which combined arnold transforming and logistic chaotic mapping for scrambling the grayscale digital image watermarking in interpolatory orthogonal multiwavelets transforming domain.

\section{The Combinational Chaotic Scrambling of Watermarking Image}

The discrete chaotic calculating based on computer is generally limited accuracy, which brings great negative impact to the performance of the chaotic system and results in dynamics degradation (Sehmitz, 2001). It has been theoritically proved that the fixed-length collection of chaotic binary sequences will constitute a group under the XOR operating (Ding \& Wang, 2009), these resulted in the multiple encrypting to be null and void when did XOR operating to the data stream with chaotic binary sequences as key for encrypting only, and the collection of two-dimensional $N \times N$ chaotic scrambled matrixes composed of a finite permutation group with the order $N \times N$ under the permutation operating. The paper proposed a scrambling algorithm which combined arnold transforming with logistic chaotic mapping, it overcomed the dynamics degradation of discrete chaotic system, trivial key space phenomenon and the single chaotic iterative trajectory being exposed, it improved the security of the scrambling algorithm also. When scrambling the digital image watermark with the combination of arnold transforming and logistic chaotic mapping, it could not only change their positions between the pixels and avoid effectively the shorter cycles of arnold transforming by the logistic chaotic mapping sequence, but also overcome the permutation group effect of the single rows or columns permutating of matrix (Jiao \& Ding, 2011).

Two-dimensional arnold mapping defined as follows:

$$
\left[\begin{array}{l}
x_{n+1} \\
y_{n+1}
\end{array}\right]=\left[\begin{array}{ll}
1 & 1 \\
1 & 2
\end{array}\right]\left[\begin{array}{l}
x_{n} \\
y_{n}
\end{array}\right](\bmod \mathrm{N})
$$

Where $x_{0}, y_{0} \in\{0,1,2, \ldots, N-1\}$. Obviously, the two-dimensional arnold mapping is chaotic mapping(Jiao \& Ding,2011), and is of cyclical phenomenon, for the scrambled watermark image by the two-dimensional arnold mapping provided that the scrambing algorithm was public, then one can make a recovery from the original image by starting to iterate in limited steps with any state of the scrambling space.

The basic logistic chaotic mapping defined as follows:

$$
x_{n+1}=\mu x_{n}\left(1-x_{n}\right)
$$

where, $n \in Z, \mu \in[0,4], x_{n} \in(0,1), \mu$ is the branch coefficient, when $3.5699456<\mu \leq 4$, the logistic chaotic mapping works in chaotic state (Li \& Hou, 2006; Sun \& Lv, 2006).

It has been proven theoretically that two different chaotic sequence $x_{0}, x_{1}, \ldots, x_{n}$ and $y_{0}, y_{1}, \ldots, y_{n}$ which generated in accordance with the formula (2) with different initial $x_{0}$ and $y_{0}$ will be zero cross-correlational, which reflects the extreme sensitivity of the logistic chaotic mapping to initial. To strengthen the security of the embedded watermark, at first, scanning the scrambled watermark image in accordance with formula (1) into an one-dimensional vector, and then, scrambling the vector by means of a same sized logistic chaotic sequence which was generated from the formula (2), thus, it avoided the short cycle of arnold transforming and the group effects of simple chaotic scrambling.

Specifically, the paper used $128 \times 128$ sized grayscale digital images to be watermarking image as shown in figure 1 , at first, scrambling the watermarking image in accordance with the formula (1), then, scanning the scrambled watermarking image into an one-dimensional vector, at last, taking a branch factor $\mu \in(3.5699456,4]$, initial seed $x_{0} \in(0,1)$ to generate a chaotic sequence in accordance with the formula (2) to scramble the vector again. The combinational scrambled watermarking image is shown in Figure 2, further experiments showed that the arnold transforming cycle of $128 \times 128$ sized images is ninety-six. 


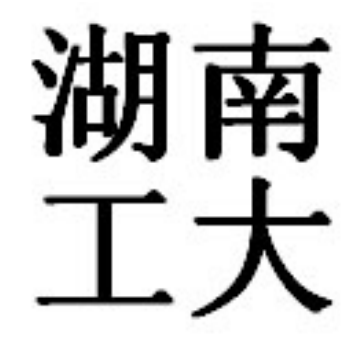

Figure 1. Watermarking image

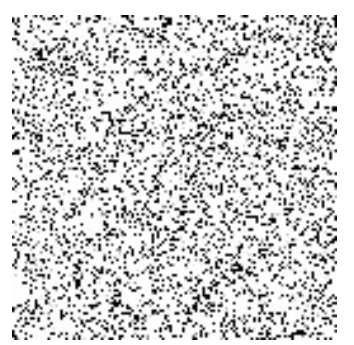

Figure 2. Combinational chaotic scrambled watermark image

\section{The Chaotic Digital Image Watermarking Algorithm of Interpolatory Orthogonal Multiwavelets Transforming Domain}

\subsection{Signal Decomposition and Reconstruction by Interpolatory Orthogonal Multiwavelets}

Suppose $\phi=\left(\phi_{0}(t), \phi_{1}(t)\right)^{T} \in\left(L^{2}(R)\right)^{2}$ to be a continuous compactly supported orthogonal multiscaling function, $\phi_{0}(t)$ and $\phi_{1}(t)$ satisfy interpolatory condition

$$
\phi_{i}\left(k+\frac{l}{2}\right)=\delta_{k, 0} \delta_{i, l}, k \in Z, i, l \in\{0,1\}
$$

and vector refinement equations of the form

$$
\phi(t)=\sum_{k \in Z} P_{k} \phi(2 t-k)
$$

where $\left\{P_{k}\right\}_{k \in Z}$ is a finitely supported sequence of $2 \times 2$ matrices. Let $\varphi=\left(\varphi_{0}(x), \varphi_{1}(x)\right)^{T}$ to be a continuous compactly supported orthogonal multiwavelets function which is generated by $\phi=\left(\phi_{0}(x), \phi_{1}(x)\right)^{T}$, then $\varphi$ has the same interpolatory property

$$
\varphi(k)=\delta_{k, 0}(1,0)^{T}, \varphi\left(k+\frac{1}{2}\right)=\delta_{k, 0}(0,1)^{\mathrm{T}}
$$

and satisfies vector refinement equations of the form

$$
\varphi(t)=\sum_{k \in Z} Q_{k} \phi(2 t-k)
$$

where $\left\{Q_{k}\right\}_{k \in Z}$ is a finitely supported sequence of $2 \times 2$ matrices.

$$
\begin{aligned}
& \forall f(t) \in L^{2}(R), \text { denoting } \phi_{i, j, n}(t)=2^{\frac{j}{2}} \phi_{i}\left(2^{j} t-n\right), \quad \varphi_{i, j, n}(t)=2^{\frac{j}{2}} \varphi_{i}\left(2^{j} t-n\right), \quad c_{i, j, n}=\left\langle f(t), \phi_{i, j, n}(t)\right\rangle \\
& =\int_{-\infty}^{\infty} f(t) \phi_{i, j, n}(t) d t, C_{j, n}=\left(c_{0, j, n}, c_{1, j, n}\right)^{T}, d_{i, j, n}=\left\langle f(t), \varphi_{i, j, n}(t)\right\rangle=\int_{-\infty}^{\infty} f(t) \varphi_{i, j, n}(t) d t, \text { and } D_{j, n}=\left(d_{0, j, n}, d_{1, j, n}\right)^{T},
\end{aligned}
$$

according to the formula (4) and (6), established the following fast decomposition and reconstruction algorithm of $f(t)$ :

$$
\begin{gathered}
C_{j, n}=\frac{1}{\sqrt{2}} \sum_{k} P_{k-2 n} C_{j+1, k} \\
D_{j, n}=\frac{1}{\sqrt{2}} \sum_{k} P_{k-2 n} C_{j+1, k} \\
C_{j+1, n}=\frac{1}{\sqrt{2}} \sum_{m} P_{n-2 m}^{T} C_{j, m}+\frac{1}{\sqrt{2}} \sum_{m} Q_{n-2 m}^{T} D_{j, m}
\end{gathered}
$$

The interpolatory property (3) tells us that $C_{0, n}$ can be exactly given by the samples of the signal $C_{0, n}=\left(c_{0,0, n}, c_{1,0, n}\right)^{T}=\left(f(n), f\left(n+\frac{1}{2}\right)\right)^{T}$, hence, lets $x(n)=f\left(\frac{n}{2}\right)$, then $C_{0, n}=(x(2 n), x(2 n+1))^{T}$, i.e. the 
advantage of interpolatory orthogonal multiple refinable functions is that the coefficients in the multiresolution representation can be realized by sampling instead of inner products.

\subsection{The Embedding Algorithm of Watermarking Image}

Assuming the carrier grayscale digital image $X$ is of the size $M \times M$, i.e. $X=\{x(m, n), 0<m, n \leq M\}$, where $x(m, n)$ is the grayscale gradation of $X$ in the pixel $(\mathrm{m}, \mathrm{n})$, the grayscale watermarking image $W$ is of the size $N \times N$, i.e. $W=\{w(m, n), 0<m, n \leq N\}$, where $w(m, n)$ is the grayscale gradation of $W$ in the pixel (m, n). Without lost of generality, assuming the size of watermarking image is less than the size of the carrier image, and is subjected to $M=2^{p} N$, where $p \in Z^{+}$and $p \geq 2$.

Because of the human eye is more sensitive to low-frequency image region than the high-frequency image region, it was said to ensure the invisibility of the watermark that the watermarking information should be embedded into the high-frequency component of the carrier image, however, every kind of image processing operating will make serious destroying to the high-frequency component of the image and result in poor robustness of the watermark image. To find a medium between the invisibility and robustness of watermark, we should embed the watermarking information into the median-frequency coefficients of the carrier image in multiwavelets transforming domain, hence, we proposed following embedding algorithm of watermarking image:

Step 1: Taking a positive integer $\mathrm{T}$, then doing $\mathrm{T}$ modul 96 times arnold transforming to the grayscale digital watermarking image $W$ in accordance with formula (1), and provided the transformed image is $\mathrm{W}$;

Step 2: Taking a branch factor $\mu$ from the interval $(3.5699456,4]$, and initial seed $x_{0}$ from the interval $(0,1)$ to generate a chaotic sequence $\left\{x_{i}, i=1,2, \ldots, N \times N\right\}$ in accordance with the logistic mapping formula (2);

Step 3: Scanning $W_{d}^{\prime}$ into an one-dimensional vector, making use of the chaotic sequence $\left\{x_{i}, i=1,2, \ldots, N \times N\right\}$ to scramble the vector, then scanning the scrambled vector in sequence into the scrambled watermarking image $W_{d}$

Step 4: Making $2^{p-1}$ layers interpolatory orthogonal multiwavelets decomposition to the carrier image $X$, (here we choose the interpolatory orthogonal multiwavelets with multiplicity two constructed in literature (Zhou, 2002), and letting the last layer's multiwavelets decomposition coefficients of block form: $\left(\begin{array}{ll}L L & H L \\ L H & H H\end{array}\right)$;

Step 5: Embedding the scrambled watermarking image $W_{d}$ into the sub-block $H L$ and $L H$ in accordance with the formula (10) and (11) respectively;

$$
\begin{gathered}
H L^{\prime}=(1-\alpha) H L+\alpha W_{d} \\
L H^{\prime}=(1-\alpha) L H+\alpha W_{d}
\end{gathered}
$$

where $\alpha \in(0,1)$ represents the embedded strength of watermarking, the bigger value of $\alpha$, the stronger the watermarking image in the watermarked image, but it will degenerate the watermarked image, Conversely, the smaller value of $\alpha$, the better visual quality of the watermarked image, but it will weaken the robustness of the watermarking image in the watermarked image at the same time, hence, the value of $\alpha$ should be involved a trade-off between invisibility and robustness of the watermarking image in the watermarked image;

Step 6: Making inverse interpolatory orthogonal multiwavelets transforming with the coefficients which have been embedded watermarking information in step 5 to get the watermarked image $X_{w}$.

\subsection{The Extracting Algorithm of Watermarking Image}

Because of extracting the watermarking image from the watermarked image is an inverse process of embedding, we proposed an extracting algorithm of digital watermarking image in accordance with the embedding algorithm of watermarking image:

Step 1: Making $2^{p-1}$ layers interpolatory orthogonal multiwavelets decomposition to the original image $X$ and the watermarked image $X_{w}$ respectively (here, $X_{w}$ has been degenerated or attacked potentially);

Step 2: Extracting two watermarking images from their sub-block $H L$ and $L H$ in accordance with the formulas (10) and (11) respectively, then, letting $W_{d}$ to be the scrambled watermarking image that is the average of the extracted two watermarking images;

Step 3: To get the transitional watermarking image $W_{d}^{\prime}$, making use of the logistic chaotic sequence 
$\left\{x_{i}, i=1,2, \ldots, N \times N\right\}$ on $W_{d}$ to do an inverse operating which is recited in step 3 of the embedding algorithm;

Setp 4: Doing 96 subtracts T modul 96 times arnold transformations on $W_{d}^{\prime}$ in accordance with formula (1) to get the watermarking image $W^{\prime}$.

\subsection{Evaluating the Algorithm}

Generally, we should evaluate the watermarking algorithm from both sides invisibility and robustness. By the contrast of the extracted watermarking image $W^{\prime}$ and the original watermarking image $W$, we can determine the degree of similarity between them visually, but such a subjective or intuitive judgement is often subject to the observer's experience, environmental conditions and so on, therefore, it's necessary to take quantitative measurement to the degree of similarity. On one hand, the normalized degree of similarity (NDS) is applied to evaluate the robustness of the watermarking algorithm (Pan \& Gao, 2002):

$$
N D S=\frac{\sum_{m, n} w^{\prime}(m, n) w(m, n)}{\sum_{m, n} w^{2}(m, n)}
$$

Clearly, $0<N D S \leq 1$, and the larger value of $N D S$, the better similarity between the original watermarking image and the extracted watermarking image, on the other hand, the peak signal to noise ratio( $P S N R)$ is applied to evaluate the degree of distortion of the watermarked image $X_{w}$ contrast with the carrier image $X$, i.e., evaluating the invisibility of the watermarking algorithm:

$$
P S N R=10 \log \frac{M \times N \times 255^{2}}{\sum_{m=1}^{M} \sum_{n=1}^{N}\left(x(m, n)-x_{w}(m, n)\right)^{2}}
$$

Generally, the larger value of PSNR, the higher fidelity of the watermarked image.

\section{Experimental Results and Analysis}

We used the $512 \times 512$ sized Lena grayscale image to be a carrier image for embedding watermarking information, shown in Figure 3, the watermarking image that was shown in Figure 1 was embedded into the carrier image in accordance with the embedding algorithm, the watermarked image is shown in Figure 4, the extracted watermarking image in accordance with the extracting algorithm from Figure 4 is shown in Figure 5. Attacking the watermarked image by cutting, median filtering, JPEG compressing and adding the additional Gaussian white noise (AGWN) respectively, where the median filter of size $3 \times 3$, the JPEG compression quality to be 10 , the AGWN variance to be 0.2 , the extracted watermarking images from the attacked watermarked images are shown in Figure 6 to Figure 9. The experimentations showed that the extracted watermarking images were still clear despite any traditional attacking, especially, the robustness of anti-JPEG compressing worked well. The numerical effects of evaluating the algorithm in accordance with the formulas (12) and (13) are shown in Table 1.

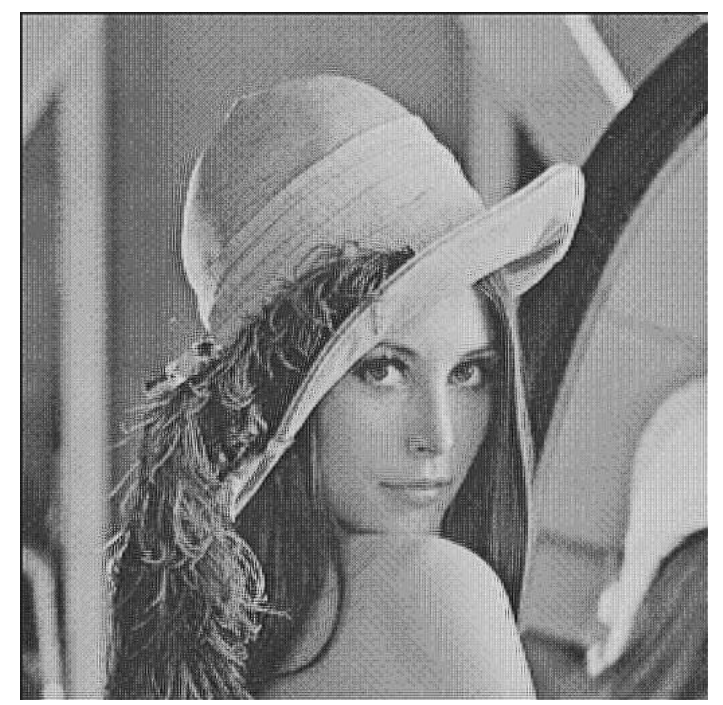

Figure 3. Lena image

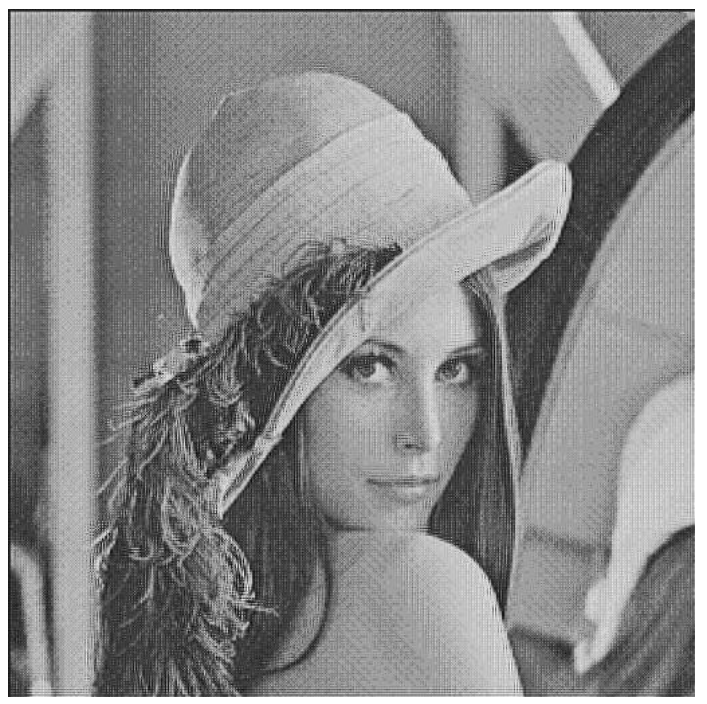

Figure 4. Watermarked image 


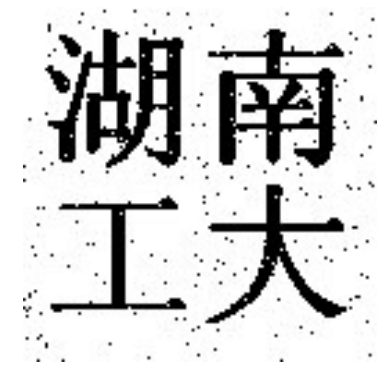

Figure 5. Extracted watermarking image

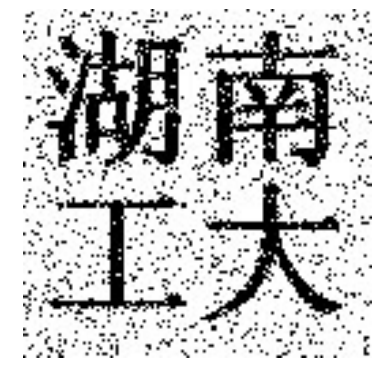

Figure 7. Extracted watermark out of median filtering attacking

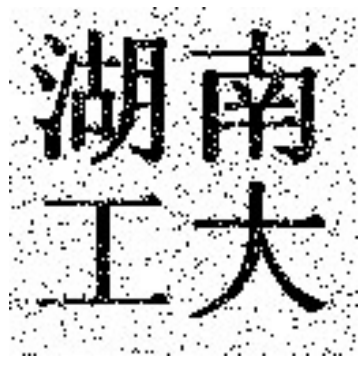

Figure 6. Extracted watermark out of cutting attacking

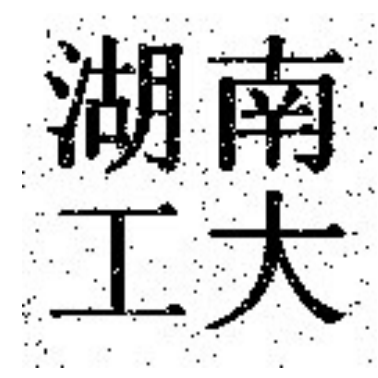

Figure 8. Extracted watermark out of JPEG compressing attacking

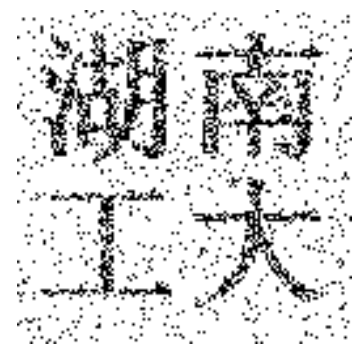

Figure 9. Extracted watermark out of AGWN attacking

Table 1. The numerical effects of evaluating

\begin{tabular}{llllll}
\hline Attacking type & PSNR & NDS & Attacking type & PSNR & NDS \\
\hline No attacking & 19.3353 & 0.98514 & Adding AGWN & 17.0865 & 0.94147 \\
Cutting & 15.6226 & 0.95082 & JPEG compressing & 19.1017 & 0.98514 \\
Median filtering & 18.2841 & 0.89772 & & & \\
\hline
\end{tabular}

\section{Results}

The experimental results showed that the characteristic of nonperiodic and pseudo-randomness of chaotic sequence improved the robustness of the watermark embedding algorithm, the combinational scrambling the watermarking image with arnold transforming and chaotic sequence improved the security of the watermark embedding algorithm, the interpolatory properties of multiwavelets enables us to find the multiresolution decomposition coefficients from the samples of the signal rather than the inner products, i.e. it improved the computational efficiency of the algorithm greatly. The experimentations showed that the quality of the watermarked image was better and there was stronger resistance against general image processing such as cutting, median filtering, JPEG compressing, noising, i.e., the watermarking algorithm has good concealment and robustness, it makes the algorithm to have better practical application value. 


\section{Acknowledgment}

The author would like to thank the editor and the reviewers for their helpful comments.

\section{References}

Chui, C. K., \& Lian, J. (1996). A Study of Orthonormal Multiwavelets. Applied Numer. Math, 20, 273-298. http://dx.doi.org/10.1016/0168-9274(95)00111-5

Ding, W. X., \& Wang, H. (2009). Theoretical and Experimental Proof That 2D Chaotic Arrays Are Permutation Groups. Journal of National University of Defense Technology, 31, 94-98.

Fan, Y. J., \& Sun, X. H. (2006). An Image-scrambling Algorithm Based on Mixed Chaotic Sequences. Journal of Image and Graphics, 11, 387-393.

Gao, F., \& Zhang, H. (2007). A Frequency-Hopping Blind Watermarking Algorithm Based on Chaotic Sequence Locating. Transactions of Beijing Institute of Technology, 27, 709-712.

Jiang, L., Huang, H., Zhang, C. F., \& Zhu, S. H. (2009). Image Zooming Through In terpolatory Orthonormal Multiwavelets. Journal of Chinese Computer Systems, 30, 106-110.

Jiao, W., \& Ding, W. X. (2011). A Digital Image Watermarking Scheme Based on Chaotic Mapping and Singular Value Decomposition. Signal Processing, 27, 1219-1223.

Li, Z. H., \& Hou, J. J. (2006). DCT-Domain Fragile Watermarking Algorithm Based on Logistic Maps. Acta Electronica Sinica, 34, 2134-2137.

Lu, W., Lu, H. T, \& Chung, F. L. (2006). Feature Based Watermarking Using Watermark Template Mateh. Applied Mathematies and Computation, 177, 377-386. http://dx.doi.org/10.1016/j.amc.2005.11.015

Pan, R., \& Gao, Y. X. (2002). Image Watermarking Method Based on Wavelet Transform. Journal of Image and Graphics (Series A), 7, 667-671.

Sehmitz, R. (2001). Use of Chaotie Dynamieal Systems in Cryptography. Journal of the Franklin Institute, 338 , 429-441. http://dx.doi.org/10.1016/S0016-0032(00)00087-9

Sun, F. Y., \& Lv, Z. W. (2006). Cryptographic spatial chaos sequence. Acta Physical Sinica, 60, 1-6.

Xia, X. G., Hardin, D. P., Geronimo, J., \& Suter, B. (1996). Design of Prefilters for Discrete Multiwavelet Transforms. IEEE Trans. on Signal Processing, 44, 25-35. http://dx.doi.org/10.1109/78.482009

Xiang, H., Wang, L. D., Lin, H., \& Shi, J. Y. (1999). Digital Watermarking System with Chaotie Sequenees. In Proeeedings of SPIE Conferenee on Seeurity and Watermarking of Multimedia Contents, 49-457.

Yang, S. Z. (2006). Compactly Supported Orthogonal Interpolation Multiwavelets. Acta Mathematica Sinica, 48, $565-572$.

Zhao, J., \& Qi, H. (2004). An Improved Wavelet Watermarking Scheme Based on Logistic Chaotic Sequences. Acta Photonica Sinica, 33, 1236-1238.

Zhou, D. X. (2002). Interpolatory Orthogonal Multiwavelets and Refinable Functions. IEEE Trans. on Signal Processing, 50, 520-527. http://dx.doi.org/10.1109/78.984728 